\title{
The nonlinear coupling between gyroradius scale turbulence and mesoscale magnetic islands in fusion plasmas
}

\author{
W.A. Hornsby, A.G. Peeters, A.P. Snodin, \\ F.J. Casson, Y. Camenen, G. Szepesi, M. Siccinio, E. Poli \\ Centre for Fusion, Space and Astrophysics, Dept. of Physics, \\ University of Warwick, Coventry, U.K. \\ Max-Planck-Institut für Plasmaphysik, Boltzmannstrasse 2, \\ D-85748 Garching bei München, Germany
}

September 28, 2010

\begin{abstract}
The interaction between small scale turbulence (of the order of the ion Larmor radius) and meso-scale magnetic islands is investigated within the gyrokinetic framework. Turbulence, driven by background temperature and density gradients, over nonlinear mode coupling, pumps energy into long wavelength modes, and can result in an electrostatic vortex mode that coincides with the magnetic island. The strength of the vortex is strongly enhanced by the modified plasma flow response connected with the change in topology, and the transport it generates can compete with the parallel motion along the perturbed magnetic field. Despite the stabilizing effect of sheared plasma flows in and around the island, the net effect of the island is a degradation of the confinement. When density and temperature gradients inside the island are below the threshold for turbulence generation, turbulent fluctuations still persist through turbulence convection and spreading. The latter mechanisms then generate a finite transport flux and, consequently, a finite pressure gradient in the island. A finite radial temperature gradient inside the island is also shown to persist due to the trapped particles, which do not move along the field around the island. In the low collisionality regime, the finite gradient in the trapped population leads to the generation of a bootstrap current, which reduces the neo-classical drive.
\end{abstract}

Magnetic islands are formed in fusion plasmas by the presence of the non-ideal instability known as the tearing mode $[1,2]$. The presence of large scale magnetic islands is severely detrimental to the confinement of the plasma and can lead to disruptions. Understanding and controlling the instability is, therefore, of profound importance to fusion experiments, especially for larger machines such as ITER[3].

The tearing mode acts to change the topology of the magnetic field through the process of reconnection, which occurs in the neighbourhood of magnetic surfaces with a rational 
magnetic winding number (safety factor) $q=m / n$, where $\mathrm{m}(\mathrm{n})$ is the poloidal (toroidal) mode number. Indeed, any rational surface is topologically unstable against a poloidal flux perturbation that is resonant with the field line winding, with the perturbation generating an island structure in the magnetic field. Of particular importance to a reactor is the neoclassical drive (the Neoclassical Tearing Mode, or NTM $[4,5]$ ) of the tearing mode. The fast transport along the magnetic field leads to a flattening of the pressure gradient inside the island, which in turn leads to a reduction of the neoclassical bootstrap current. This perturbation in the current density has the same helicity as the island and drives the tearing mode unstable. NTMs are expected to limit the pressure gradient at which a reactor can operate and their stability condition is therefore an active area of research.

The neoclassical tearing mode is often observed to have a threshold behaviour, i.e. the mode only grows when the island size exceeds a threshold width. Two main candidates have been proposed to explain the threshold: the polarization current connected with the island rotation [6,7], and the influence of the finite radial transport on the pressure gradient inside the island [8]. This paper will largely deal with the latter mechanism. Radial transport of heat and particles will try to maintain the pressure gradient inside the island against the profile flattening due to the fast motion along the magnetic field. For sufficiently small islands the perpendicular transport dominates, preventing the pressure profile flattening and, therefore, eliminating the neoclassical drive. The temperature profile under the influence of both perpendicular as well as parallel transport has been modelled in the literature [8] by the steady-state heat transport equation

$$
D_{\|} \nabla_{\|}^{2} T+D_{\perp} \nabla_{\perp}^{2} T=0
$$

where $D_{\|}$and $D_{\perp}$ are the heat conduction coefficients parallel and perpendicular to the perturbed field lines respectively, and are assumed to be time and spatially constant.

The perpendicular transport in the Eq. (1) is widely acknowledged to be due to gyroradius scale turbulence. Microinstabilities, driven by temperature and density gradients, lead to the generation of this turbulence, which is regulated by zonal flows. The latter flow patterns in turn are generated through the nonlinear interaction between the turbulent eddies. This naturally raises many questions on the modelling of the perpendicular transport. When turbulence is driven by the gradients, the diffusion coefficient is a function of these, making the heat equation nonlinear in the gradients. While there is in general some scale separation between turbulence and the island size, it is not obvious that the scale separation is large enough to model the perpendicular transport through a diffusion equation. Turbulence might generate structures of the size of the island, which transport heat through convection.

The interaction of magnetic islands and turbulence has recently received much interest. Turbulence has been shown to modify the stability and dynamics of the tearing mode [9, 10, 11, 12]. Furthermore, the influence of mesoscale magnetic island structures on small scale turbulence (and vice versa) is expected to affect heat and particle transport properties [13, $14,15]$. The self-consistent electrostatic response connected with the island may attain large gradients resulting in a considerable sheared plasma flow. Such flows are known to stabilize small scale turbulence by the shearing apart of eddy structures [16, 17] and, therefore, an energy confinement improvement may result as a consequence of a magnetic island. Indeed there is evidence that steep density and temperature gradients can form in the vicinity of rational surfaces [18]. However, it is unclear if the shearing is large enough such that the reduction in transport can compensate the increase in the radial heat flux due to the perturbed radial magnetic field of the island.

Presented is a study of turbulence in the presence of a static island, an approximation that is valid due to the large time scale seperation between the turbulence and the magnetic 
island. In this paper, the Vlasov flux-tube code GKW[20, 21] is employed and the full (kinetic) electron dynamics is retained. An analogous approach has been employed in Ref.[19], where the turbulence in the presence of a static island has been studied by means of global particle-in-cell simulations with adiabatic electrons. The similarities and the differences between the approach used here and that of Ref.[19] are addressed in the conclusions.

This paper is structured as follows. Section 1 describes the gyrokinetic model used. Results are presented in Sections 3, 4 and 5, and conclusions are drawn in Sec.6.

\section{Theoretical Framework}

The influence of a magnetic island on the turbulence is studied using the gyrokinetic framework, with numerical solutions obtained using a modified version of the gyrokinetic flux-tube code GKW of which more details can be found in $[20,21]$. The delta- $f$ approximation is used, in which the distribution function is split into a background $F$ and a perturbed distribution $f$. The final equation for the perturbed distribution function $f$ can be written in the form

$$
\frac{\partial g}{\partial t}+\left(v_{\|} \mathbf{b}+\mathbf{v}_{D}\right) \cdot \nabla f+\mathbf{v}_{\chi} \cdot \nabla g-\frac{\mu B}{m} \frac{\mathbf{B} \cdot \nabla B}{B^{2}} \frac{\partial f}{\partial v_{\|}}=S,
$$

where $S$ is the source term which is determined by the background distribution function, $\mu$ is the magnetic moment, $v_{\|}$is the velocity along the magnetic field, $B$ is the magnetic field strength, $\mathrm{m}$ and $\mathrm{Z}$ are the particle mass and charge number respectively. Here, $g=$ $f+(Z e / T) v_{\|}\left\langle A_{\|}\right\rangle F_{m}$ is used to absorb the time derivative of the parallel vector potential $\partial A_{\|} / \partial t$ which enters the equations through Ampères law. The background is assumed to be a Maxwellian $\left(F_{M}\right)$, with particle density $(n)$ and temperature $(T)$

$$
F=F_{M}=\frac{n}{\pi^{3 / 2} v_{\mathrm{th}}^{3}} \exp \left[-\frac{v_{\|}^{2}+2 \mu B / m}{v_{\mathrm{th}}^{2}}\right],
$$

which determines the source term

$$
\begin{aligned}
S= & -\left(\mathbf{v}_{\chi}+\mathbf{v}_{D}\right) \cdot\left[\frac{\nabla n}{n}+\left(\frac{v_{\|}^{2}}{v_{\mathrm{th}}^{2}}+\frac{\mu B}{T}-\frac{3}{2}\right) \frac{\nabla T}{T}\right] F_{M} \\
& -\frac{Z e}{T}\left[v_{\|} \mathbf{b}+\mathbf{v}_{D}\right] \cdot \nabla\langle\phi\rangle F_{M} .
\end{aligned}
$$

All equilibrium quantities, such as the temperature and density, are assumed to be functions of the radius only. The thermal velocity $v_{\text {th }} \equiv \sqrt{2 T / m}$, and the major radius $(R)$ are use to normalise the length and time scales. Using standard gyrokinetic ordering, the length scale of perturbations along the field line $\left(R \nabla_{\|} \approx 1\right)$ are significantly longer than those perpendicular to the field $\left(R \nabla_{\perp} \approx 1 / \rho_{*}\right)$. Here, $\rho_{*}=\rho_{i} / R$ is the normalised ion Larmor radius (where $\rho_{i}=m v_{t h} / e B$ and $v_{t h}=\sqrt{2 T_{i} / m_{i}}$ ).

The velocities in Eq.(2) are from left to right: the parallel motion along the unperturbed field $\left(v_{\|} \mathbf{b}\right)$, the drift motion due to the inhomogeneous field $\left(\mathbf{v}_{D}\right)$, and the motion due to the perturbed electromagnetic field $\left(\mathbf{v}_{\chi}\right)$. The drift due to the inhomogeneous magnetic field can be written in the form $[21]$

$$
\mathbf{v}_{D}=\frac{1}{Z e}\left[\frac{m v_{\|}^{2}}{B}+\mu\right] \frac{\mathbf{B} \times \nabla B}{B^{2}}+\frac{m v_{\|}^{2}}{2 Z e B} \beta^{\prime} \mathbf{b} \times \nabla \psi,
$$




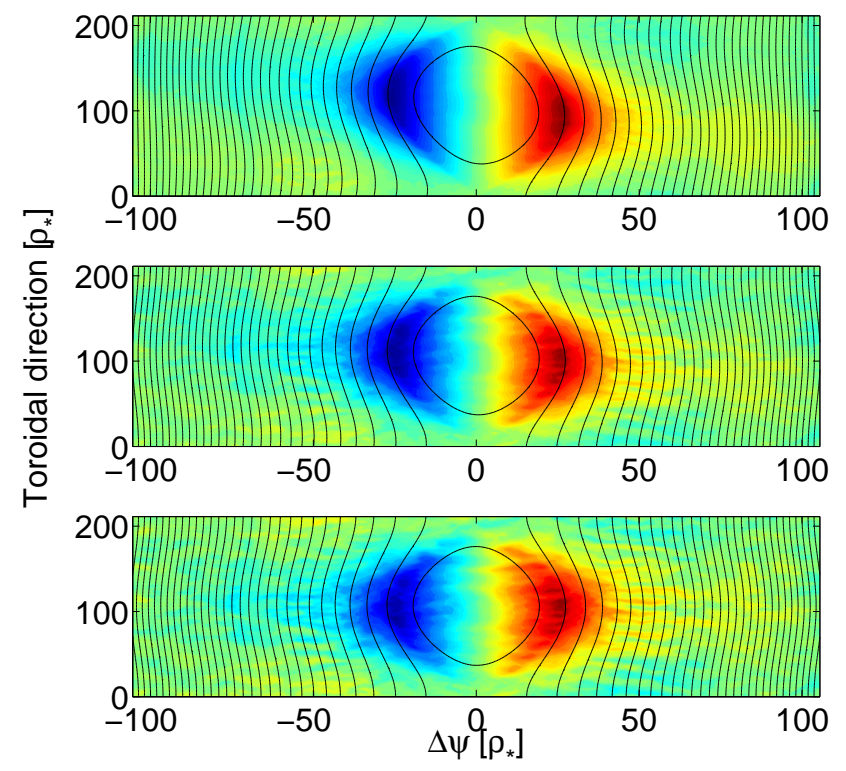

Figure 1: (Color online) The perturbed electron temperature at (top) $\theta=\pi$, high field side, (middle) $\theta=\pi / 2$ and (bottom) $\theta=0$, low field side. The localisation of turbulence to the outboard side is evident, also evident is the skewing of the flux tube due to the magnetic shear.

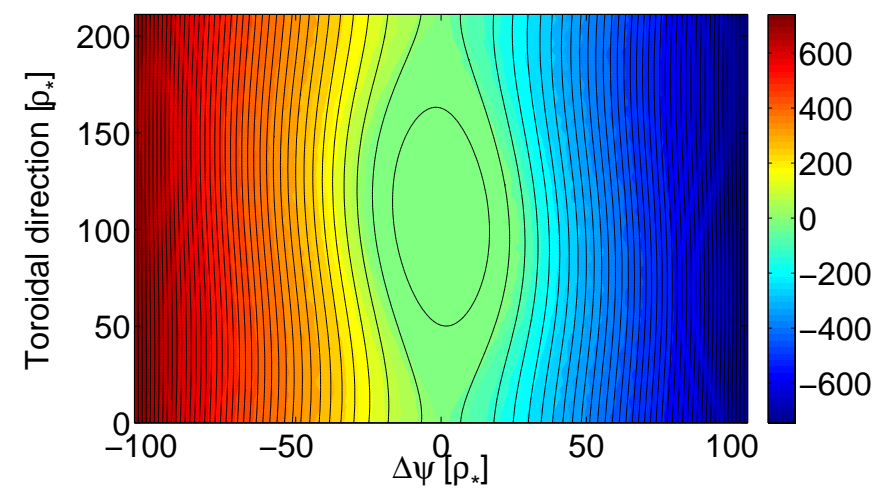

Figure 2: (Color online) The total equilibrium electron temperature at the high field side showing the self-consistent flattening of the temperature profile within the island separatrix. Temperatures are relative to the reference temperature at the centre of the domain. 
whereas the motion due to the perturbed electromagnetic field

$$
\mathbf{v}_{\chi}=\frac{\mathbf{b} \times \nabla \chi}{B},
$$

is the combination of the $E \times B$ velocity $\left(\mathbf{v}_{E}=\mathbf{b} \times \nabla\langle\phi\rangle / B\right)$ and the parallel motion along the perturbed field line $\left(\mathbf{v}_{\delta B}=-\mathbf{b} \times \nabla v_{\|}\left\langle A_{\|}\right\rangle / B\right)$. These two effects are combined into one velocity through the definition of a new field $\chi=\langle\phi\rangle-v_{\|}\left\langle A_{\|}\right\rangle$. Here, the angled brackets denote gyro-averaged quantities.

The electrostatic potential is calculated from the gyrokinetic Poisson equation which in Fourier space is

$$
\begin{array}{r}
\sum_{s p} Z_{s p} n_{R s p}\left[2 \pi B \int \mathrm{d} v_{\|} \mathrm{d} \mu J_{0}\left(k_{\perp} \rho_{s p}\right) \hat{g}_{s p}+\right. \\
\left.\frac{Z_{s p}}{T_{R s p}}\left[\Gamma\left(b_{s p}\right)-1\right] \hat{\phi}\right]=0,
\end{array}
$$

where $b=\frac{1}{2} m_{R} T_{R}\left(k_{\perp} \rho_{*} R_{\text {ref }} / Z B^{2}\right)^{2}=\frac{1}{2} \frac{k_{\perp}^{2} m^{2} v_{\text {th }}^{2}}{e^{2} B^{2}}, k_{\perp}$ being the perpendicular wavenumber and $J_{0}$ are zeroth order Bessel functions of the first kind.

The timescale of the generation and evolution of drift wave turbulence in plasmas is short compared with evolution of the magnetic island structure. Consequently, the island can be taken to be static during the simulation and its initialisation is described below. The perturbed vector potential is artificially imposed in the simulations. Since $\hat{A}_{\|}$is time independent the only effect it generates is the parallel motion along the perturbed field $v_{\|} \delta \mathbf{B} / B=v_{\|} \mathbf{b} \times \nabla\left\langle A_{\|}\right\rangle / B$. The vector potential is imposed and only the Poisson equation (quasi-neutrality) is retained. Ampères law is not solved for, however, at the scales of interest the time evolution of the island is very slow and is thus neglected. The turbulence is essentially electrostatic with no magnetic perturbations other than that of the island.

The island structure is introduced in the model through an imposed perturbed vector potential parallel to the background magnetic field with the form:

$$
A_{\|}=\tilde{A}_{\|} \exp [\mathrm{i}(m \theta-n \phi)]
$$

where $\theta$ is the poloidal angle, $\phi$ is the toroidal angle, and $\tilde{A}_{\|}$is the amplitude of the perturbation. This is often referred to as the constant- $\psi$ approximation. GKW uses straight field line Hamada coordinates $(s, \zeta, \psi)$ where $s$ is the coordinate along the magnetic field and $\zeta$ is the generalized toroidal angle. For circular concentric surfaces, the transformation of poloidal and toroidal angle to these coordinates is given by $[21](s, \zeta)=(\theta / 2 \pi,[q \theta-\phi] / 2 \pi)$. Assuming the winding of the magnetic field is resonant $(q=m / n)$ in the center of the computational domain $\left(\psi_{0}=r_{0} / R=\epsilon\right.$, where $r$ is the radius of the magnetic surface, and $R$ is the distance of the centre of the surface to the axis of symmetry) and expanding $q$ up to first order in $\Delta \psi(\Delta \psi$ being the radial distance from the resonant surface), $q=m / n+\Delta \psi(\partial q / \partial \psi)$ then yields

$$
A_{\|}=\tilde{A}_{\|} \exp [2 \pi \mathrm{in}(\zeta-s \partial q / \partial \psi \Delta \psi)] .
$$

The wave vector of the island is $k_{\zeta}^{I} \rho_{i}=2 \pi n \rho_{*}$. GKW uses a Fourier representation in the plane perpendicular to the magnetic field. The periodicity constraint on the torus shaped magnetic surface then dictates a relation between the radial and toroidal modes. In the case with an island structure this constraint can be expressed as

$$
k_{\psi}=(p / N) k_{\zeta}^{I} \frac{\partial q}{\partial \psi}=(p / N) 2 \pi n \rho_{*} \frac{\partial q}{\partial \psi},
$$



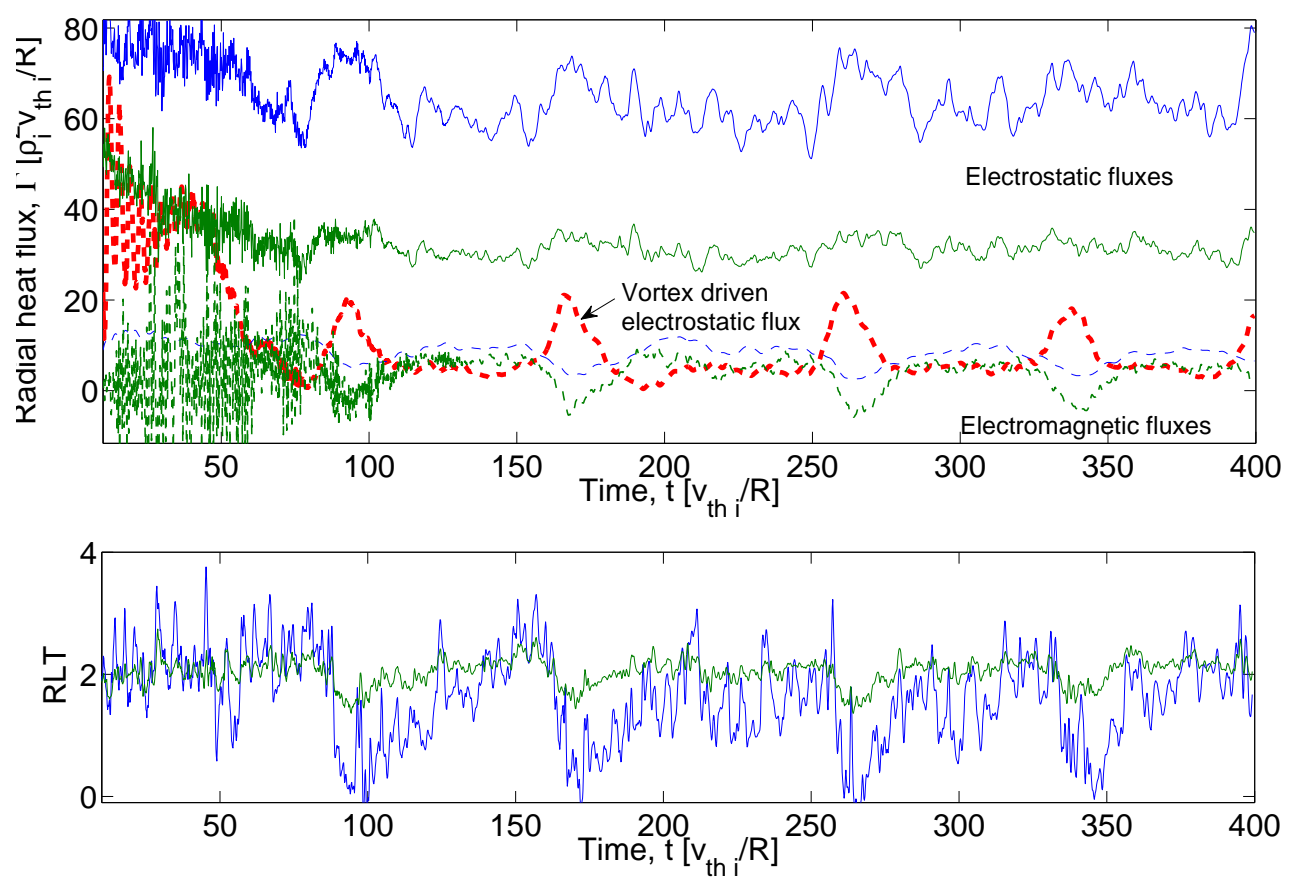

Figure 3: (Color online) Top: time traces of the total electrostatic (full lines) and magnetic flutter (dashed thin lines) turbulent heat fluxes integrated over the whole simulation volume (for case LRb) for ions (blue lines) and electrons (green lines) during the converged stage of a simulation with a magnetic island width of $w=24 \rho^{*}$. The thick red dotted line gives the electrostatic heat flux generated by the modes with the same poloidal wavelength as the island. Bottom: Time traces of the normalised average electron (green line) and ion (blue line) density gradients at the O-point. The drop in density gradients is a measure of the strength of the potential vortex within the island. 
where $p$ and $N$ are an integers. $N$ is the integer that determines the radial mode spacing

$$
\Delta k_{\psi}=(1 / N) k_{\zeta}^{I} d q / d \psi .
$$

Using $p=1$ one directly obtains the radial size of the box

$$
\Delta \psi \rightarrow\left[-\frac{N}{2 n \partial q / \partial \psi}, \frac{N}{2 n \partial q / \partial \psi}\right]
$$

The Fourier amplitudes (denoted by hatted symbols) of the perturbed vector potential can be calculated by integrating over the radial domain to obtain

$$
\begin{array}{r}
\hat{A}\left(k_{\psi}, k_{\zeta}, s\right)=C \int \mathrm{d} \Delta \psi \exp \left[-2 \pi \mathrm{in} \frac{\partial q}{\partial \psi} \psi[p+s]\right] \\
=\frac{C N}{\pi n \partial q / \partial \psi} \frac{\sin [\pi(p+N s)]}{p+N s} .
\end{array}
$$

The expression for the vector potential can be expanded in the Fourier modes used for the numerical implementation

$$
\begin{aligned}
\hat{A}\left(s, k_{\zeta}, k_{\psi}\right) & =\tilde{A}_{\|} N \exp \left(-\imath k_{\zeta}^{I} \zeta\right) \sum_{p=-\infty}^{\infty} \exp \left(-\frac{(s N+p)^{2}}{L^{2}}\right) \\
& \times \frac{\sin (\pi(s N+p))}{\pi(s N+p)} \exp \left(\imath p k_{\psi} \Delta \psi\right),
\end{aligned}
$$

This expression contains an additional ad-hoc damping at higher radial mode numbers (the term $\left.\exp \left[-(s N+p)^{2} / L^{2}\right]\right)$ in order to prevent the appearance of unphysical discontinuities in the vector potential which forms the island. These may form because, in general the island is not periodic within the sheared flux tube domain when represented by fourier harmonics.

The results are unaffected provided the scale length $L$ is chosen to be large enough. The contour lines of the perturbed vector potential given above summed with the background $A_{\| 0}=R B_{0} \Delta \psi^{2} / 2 q_{0}(1+\hat{s})$ form the surfaces traced out by the magnetic field and exhibit an island structure in the plane perpendicular to the field. These perturbed flux surfaces are shown in Fig.1,2. Note that the radial asymmetry in the island is due to the shearing of the computational domain caused by the sheared magnetic field in combination with the field aligned coordinates. It is the computational domain that is sheared (but plotted as a square box) rather than the island. The half width of the island is defined by

$$
w=2 \sqrt{q \tilde{\Psi} / \hat{s} R B},
$$

(where $\hat{s}=(1 / q) \partial q / \partial \psi$ is the magnetic shear) and the perturbed magnetic flux, $\tilde{\Psi}$, is related to the perturbation of the parallel vector potential by the relation,

$$
\tilde{\Psi}=R A_{\|}
$$

Unless explicitly denoted otherwise, all heat fluxes are calculated as volume averaged (denoted by the \{\} brackets) guiding centre fluxes and are given by the equation

$$
Q_{i}^{\alpha}=\left\{\int \mathrm{d}^{3} \mathbf{v} \tilde{\mathbf{v}}_{E} \cdot \nabla \alpha \frac{1}{2} m_{i} v^{2} f_{i}\right\}
$$


for the electrostatic fluxes, where $\tilde{\mathbf{v}}_{E}$ is the $E \times B$ velocity. The radial heat flux is obtained for $\alpha=\psi$, whereas the poloidal flux is obtained with $\alpha=\zeta$. Similarly, the heat flux due to the motion along the perturbed field lines, otherwise known as the magnetic flutter, is given by

$$
Q_{i}^{\alpha}=\left\{\int \mathrm{d}^{3} \mathbf{v} \tilde{\mathbf{v}}_{\delta B} \cdot \nabla \alpha \frac{1}{2} m_{i} v^{2} f_{i}\right\}
$$

in which $\tilde{\mathbf{v}}_{\delta B}=-\mathbf{b} v_{\|} \times \nabla\left\langle A_{\|}\right\rangle / B$.

\section{Simulation parameters}

The parameters used for these simulations are similar (but not equivalent) to those of the cyclone base case [23], namely:

- Normalized ion temperature gradient $R / L_{T}=6.9$

- Normalized density gradient $R / L_{N}=2.2$

- Inverse aspect ratio $\epsilon=0.19$

- Electron to ion temperature ratio $T_{e} / T_{i}=1$

- Safety factor $q=1.5$ and magnetic shear $\hat{s}=0.16$.

Presented here are the results from three different sets of simulations, which, unless stated otherwise, keep the kinetic electron effects with the true mass ratio of a Deuterium plasma. The three sets of simulations vary in resolution and the number of poloidal modes. The wave vector of the island is always the longest wavelength on the grid and a larger number of poloidal modes implies a larger scale separation between the turbulence and the island. The number of positive poloidal modes (only positive wave vectors counted), and radial modes (positive and negative) with their corresponding minimum wavenumbers and mode spacing are as follows:

- (LRa) 21 toroidal modes, 101 radial modes, $\Delta k_{\psi} \rho_{i}=0.05, \Delta k_{\zeta} \rho_{i}=0.05, k_{\zeta}^{I} \rho_{i}=0.05$

- (LRb) 21 toroidal modes, 167 radial modes, $\Delta k_{\psi} \rho_{i}=0.025, \Delta k_{\zeta} \rho_{i}=0.05, k_{\zeta}^{I} \rho_{i}=0.05$

- (HR) 41 toroidal modes, 167 radial modes, $\Delta k_{\psi} \rho_{i}=0.025, \Delta k_{\zeta} \rho_{i}=0.025, k_{\zeta}^{I} \rho_{i}=$ 0.025

The first two sets of simulations will be labelled as LRa and LRb respectively in the rest of this paper, while the third set shall be denoted by HR, the difference in nomenclature being related to the increase in poloidal resolution. Varying the poloidal resolution of the simulations, and thus the scale separation between the mode representing the island and the length scales of the turbulence has a subtle effect on the physics that will be described in the next section. For an idea of the typical size of an island, consider a $m=3, n=2$ island which is resonant at $q=1.5$. This choice effectively determines $\left.\rho_{*}=4 \cdot 10^{-3}\right)$ in the LR cases and $\rho_{*}=2 \cdot 10^{-3}$ ) in the HR case, values that correspond to a medium-size tokamak such as ASDEX Upgrade[24]. 

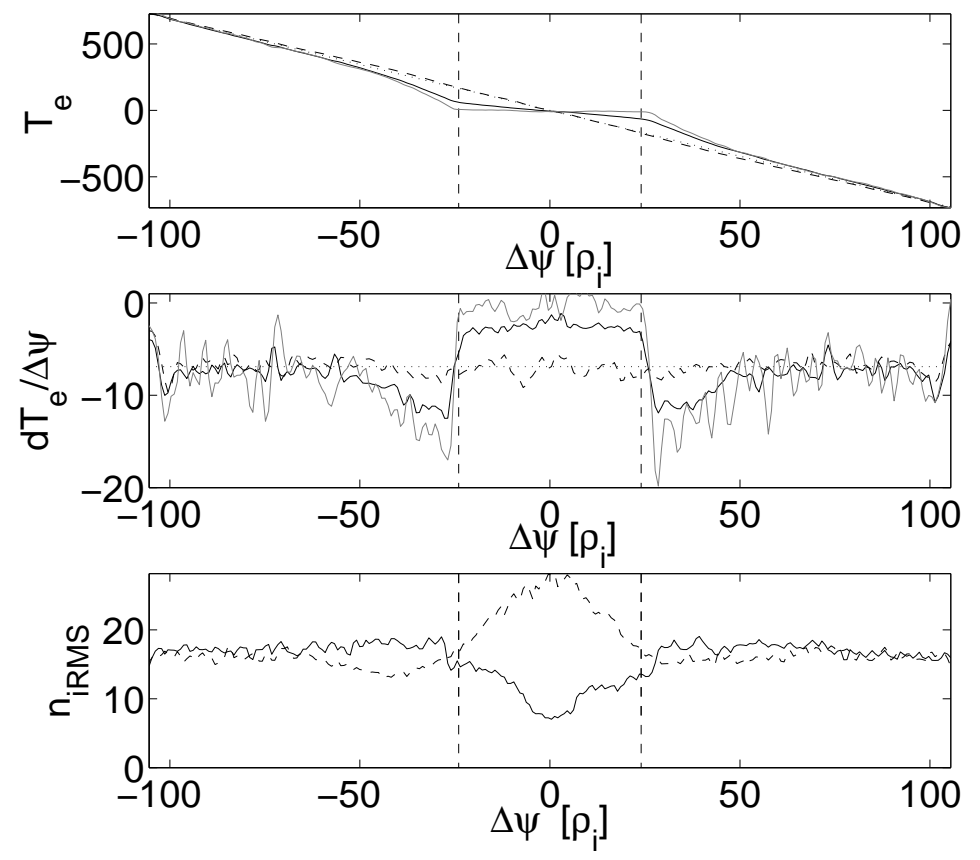

Figure 4: (for the HH case) Top panel shows the radial electron temperature profiles, black: Low field side, gray: High field side. Middle panel shows the corresponding electron temperature gradients, black: Low field side, gray: High field side.. Bottom panel shows the RMS density fluctuation profile showing a decay of the fluctuation strength within the island. Dashed lines represent lines through the $\mathrm{X}$-point in all three panels. 


\section{The effect on turbulence, zonal flows and radial heat flux}

The simulations are initialised with a small perturbed distribution, the vector potential that corresponds to an island of a chosen size, and a background distribution which has a temperature and density that is constant on the unperturbed flux surfaces. The initial density and temperature profiles are not in equilibrium in the presence of the island, and the initial stage of the simulations is characterised by large particle and heat fluxes along the perturbed magnetic field, and a flattening of the density and temperature gradients inside the island as is shown as both a perturbation (Fig. 1) and as a total temperature in Fig. 2. This initial stage shows relatively large fluctuations in all perturbed quantities, as is shown in Fig. 3

It is on top of this modified equilibrium, which is allowed to also dynamically evolve, that the turbulence is generated. The equilibrium gradients are modified in the presence of the magnetic island, with a significant flattening within the island as shown in Fig. 2 and a steepening of the gradients just outside the separatrix (see Fig.4). Fig. 4 shows the turbulent fluctuations of the density $\delta n / n_{0}$. Despite the strong increase in the gradient just outside the island, there is no corresponding increase in the turbulent fluctuations. The sheared flows around the island separatrix stabilize the turbulence as previously reported [19], and allow for the steeper gradients. Indeed, it has been suggested that sheared plasma flows connected with the island can stabilize turbulence and lead to improved confinement. However, the flattening of the profiles due to the fast parallel transport along the perturbed field lines leads to a degradation of confinement. The left panel of Fig. 8 shows that the time integrated total heat flux increases with the island size. Although, as indicated by the horizontal lines, there might be a critical width above which this effect sets in, the error bars, which are calculated as the standard deviation, do not allow for a confirmation of this critical size. TSince the perturbed distribution function is radially periodic, the temperature difference across the box is determined by the background temperature gradient and is independent of the island width. The increase in the heat flux, therefore, directly shows that confinement is degrading with increasing island width.

Turbulence is reduced within the island as the temperature and density gradients are reduced. Stronger turbulence is driven outside the island which is evident by looking at the radial flux profile around the island (Fig.5) and the radial profile of density fluctuations seen in the bottom panel of Fig. 4. However, these measures do not drop to zero within the island, implying that turbulence is able to enter the island, or can be driven within the separatrix. Linear calculations performed using the modified equilibrium gradients $\left(k_{\theta} \rho_{i}=0.42\right.$, $R / L_{n}=0.5$, electron $R / L_{T}=2.0$ ) inside the island are below the threshold for microinstabilities in the LR case, while they are marginally above the stability threshold in the HR case (a critical ion temperature gradientof $R / L_{T}=2.75$ is obtained for these parameters). When comparing simulations $\mathrm{LRb}$ and $\mathrm{HR}$, the density and electron temperature profiles are the same. However, there is a difference in the equilibrium ion temperature profile as shown in Fig. 6.

The difference between the LR and HR simulations can be explained by comparing the timescales of the parallel streaming and perpendicular transport. The timescales for a particle of species, s, to traverse the field around the island $\left(\tau_{s \|}\right)$ by assuming that transport is advective and the field line has a length

$$
L_{\|}=\frac{4 \pi q R}{m w \hat{s}} .
$$


can be estimated to be

$$
\frac{\tau_{s \|} v_{t h i}}{R}=\sqrt{\frac{T_{i} m_{s}}{T_{s} m_{i}}} \frac{4 \pi q}{\left(k_{\theta}^{I} \rho\right) \hat{s}(w / \rho)}
$$

The timescale for perpendicular transport across the island $\tau_{s \perp}$ is assumed to be diffusive

$$
\frac{\tau_{s \perp} v_{t h i}}{R}=\frac{1}{2 D_{s}^{N}}\left(\frac{w}{\rho_{i}}\right)^{2}
$$

where $D_{s}^{N}$ is the relevant transport coefficient in gyro-Bohm units $\left(D_{s}=D_{s}^{N} \rho_{i}^{2} v_{t h i} / R\right)$. For the parameters used in this paper, $\tau_{i \|} \approx 196$ for the HR case and $\tau_{i \|} \approx 97$ for the LR cases; $\tau_{e \|} \approx 3.3$ for the HR case and $\tau_{e \|} \approx 1.6$ for the LR cases, $\tau_{i \perp} \approx 40$, and $\tau_{e \perp}=80$ (times are given in of $R / v_{t h i}$ ). From the comparison of the timescales one would expect a moderate flattening for the ions and a complete flattening of the electron temperature profile. The disparity in the ion temperature gradient between the two cases can be explained by observing that the time scale for parallel heat transport is a function of the island wavevector, as such, the time scale is larger for the $H R$ case than that for LR case. In the HR case the perpendicular turbulent transport is more significant compared to the parallel heat transport which results in a larger temperature gradient. Finally, it is noted that the radial electron temperature gradient, which is expected to be flat from the timescale comparison, is in fact observed to be finite (see Fig. 4). This phenomenon will be discussed towards the end of this paper.

In the HR but also in the LR case a finite fluctuation amplitude within the island is observed. In the LR case this turbulence can not be driven by the local temperature and density gradient, and we conclude that these turbulence fluctuations are generated by the gradients outside the island separatrix. The turbulence can then move into the island through a mechanism known as turbulence spreading[25, 26, 27, 28, 31, 29]. Turbulence spreading is the mechanism by which eddies through their mutual interaction propagate from the region where they are excited into a region where they may no longer be driven, or even suppressed. Fig. 5 shows the time averaged radial heat flux in a slice through the flux tube showing both the X-points and O-points. As expected the heat mainly flows via the X-points and is largely suppressed at the O-point location. An interesting point is the poloidal asymmetry about the $\mathrm{X}$-point that is present in the heat flux, and by proxy, the turbulence within the island. This suggests the mechanism by which the turbulence moves from the X-point is linked to the particle drifts with eddies able to convect through the island in the drift direction (top to bottom) but not against it (bottom to top). This turbulence convection is different to the mechanisms suggested in the literature which are diffusive in nature[29]. The latter diffusive mechanism may still play a role, but a substantial part of the fluctuations inside the island are due to turbulence convection rather than spreading.

The heat conduction coefficients have been found to roughly follow the dependence of $\delta n / n$ and, consequently, transport in the island is reduced compared with the outer region. Finally, note that while the transport coefficients are reduced in the O-point, they are strongly enhanced at the X-point. This effect is evident in the profiles of the average density fluctuation in chords through the O-point and the X-point seen in the bottom panel of Fig 4, where the root mean square fluctuation decays through the O-point, but is enhanced through the X-point.

\section{Large scale potential structures}

Another striking observation is the formation of a large scale potential structure within the island as shown in Fig. 7. Linearly these modes $\left(k_{\theta} \rho_{i}=0.05 / 0.025\right)$ are stable even 


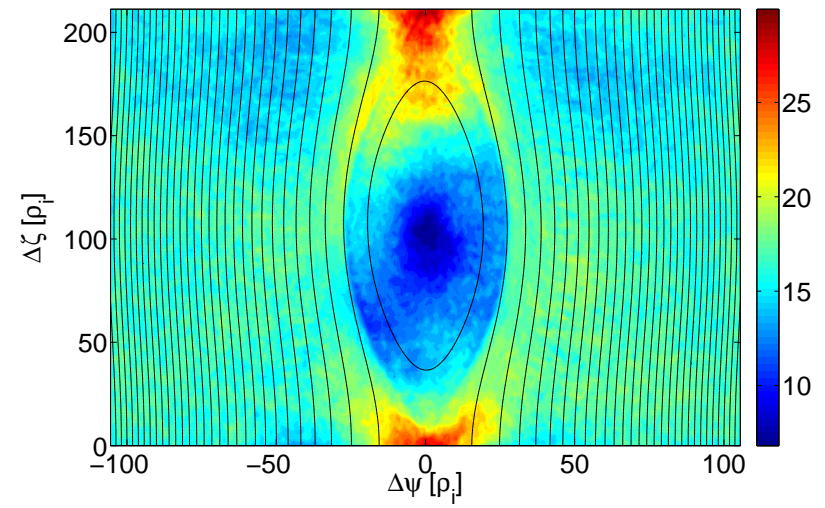

Figure 5: (Color online) The radial heat flux in a slice through the flux tube at the low field side of the torus for case LR
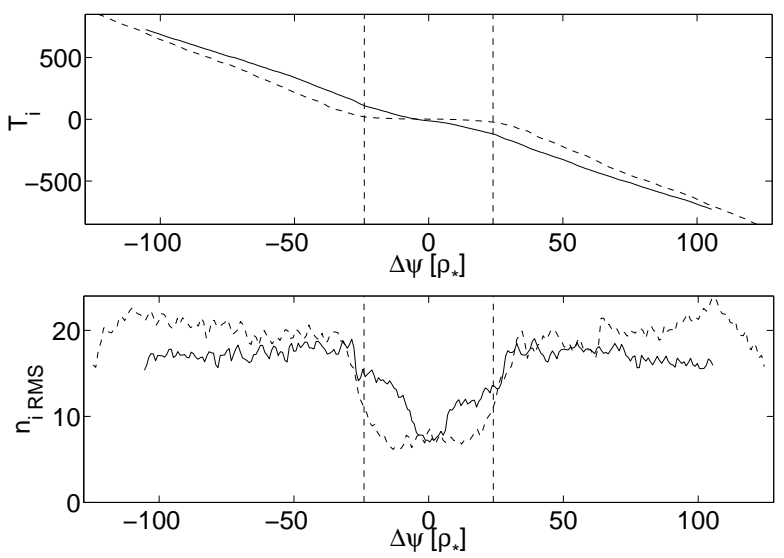

Figure 6: Bottom panel: Root mean square ion density fluctuations through the O-points for simulation runs LR (dashed) and HH (solid). Top panel: The corresponding ion temperature profiles through the O-points. 

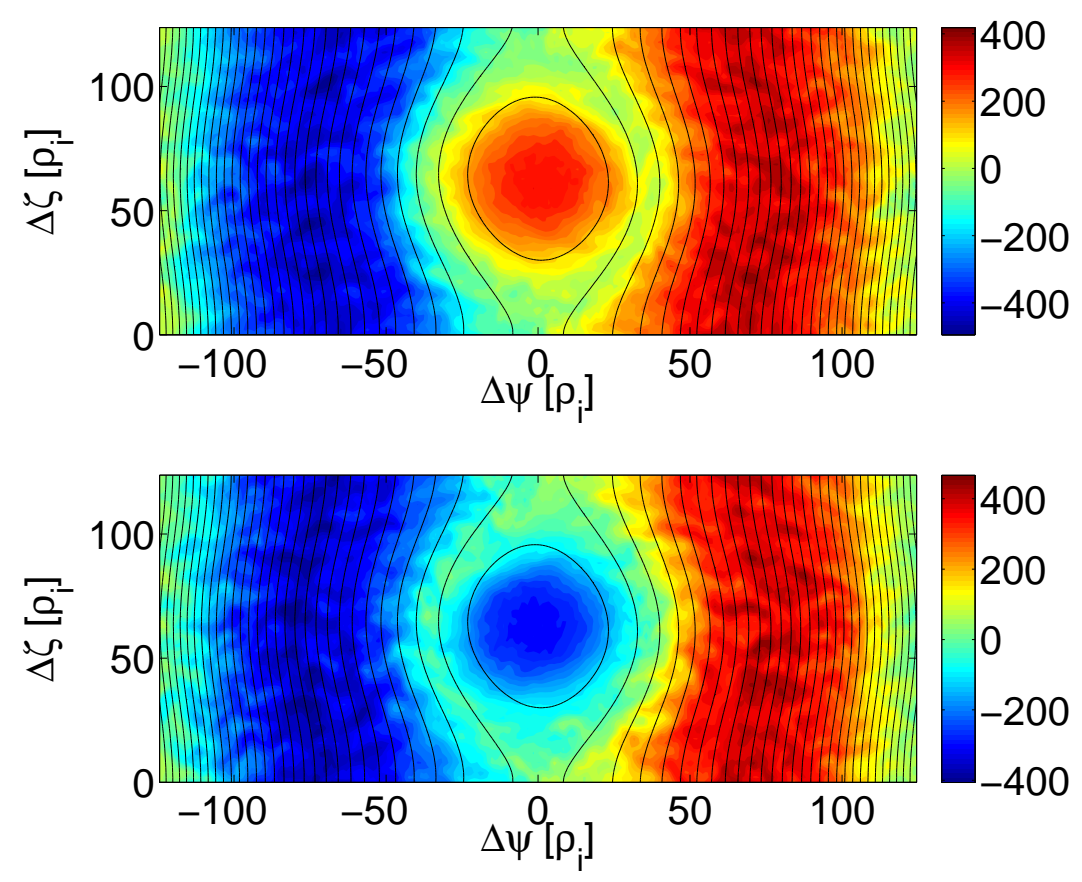

Figure 7: (Color online) Normalized electrostatic potential $\left(\phi_{N}=e \phi / T \rho_{*}\right.$, case LRb, $W / \rho_{i}=24$ ) in the plane perpendicular to the magnetic field (outboard midplane). Black lines represent the perturbed flux surfaces calculated from the total parallel vector potential. The presence of the island embedded in the turbulence not only generates flows around the island structure but also large scale electrostatic potential structures within the island which fluctuate in amplitude and sign. The top panel shows a vortex with a positive sign, while the lower panel shows that the vortex has flipped sign at a point later in the simulation. 
with the equilibrium gradients. The formation of these large scale structures is due to the nonlinear pumping by the turbulence, a process which is analogous to the mechanism for the generation of zonal flows. In the case without an island, the magnitude of the potential for the $n=0$ 'zonal' mode is largely enhanced because the electrons can not balance the ion charge through a motion along the field. Similarly, in the case with an island the parallel electron motion can not balance an ion density that is constant along the perturbed magnetic surfaces. The result is a strong enhancement of the potential perturbation which is constant on the perturbed surface and, consequently, a large ExB flow along the perturbed surfaces. Indeed, simulations with an adiabatic electron density response $n_{e}=e n_{0} \phi / T_{e}$, which lack the physics of the stronger potential response, do not show a large vortex structure. Simulations in Ref.[19], which also treat the electron response adiabatically, also fail to see a large scale vortex which further enforces the need for a fully kinetic approach.

Unlike the zonal flow that forms in the absence of the island, the velocity pattern of the vortex mode does have a radial component, and can lead to the radial transport of heat. The contribution to the electrostatic heat flux of the mode with a poloidal wave vector equal to that of the island is observed to increase with the island size as shown in Fig. 9, and is enhanced by one to two orders of magnitude compared with the case with small island. For larger island sizes most of the heat can be transported through the vortex mode.

The top panel of Fig. 3 shows the total radial heat flux as well as the radial heat flux driven by the vortex mode as a function of time. The flux associated with the mode is an indirect measure of the mode amplitude because the large convective cell, when strong, advects heat around the island and thus increases the effective heat flux. It can be seen that the amplitude of the vortex mode is not a constant in time and the direction of rotation around the vortex can flip sign (see Fig. 7). One can note a clear anti-correlation between the radial heat flux generated by the vortex and the 'magnetic flutter', i.e. the heat flux due to the motion along the perturbed magnetic field that forms the island. At its maximum strength the transport due to the vortex largely exceeds the flutter flux, and on average its contributions to the heat flux is comparable. (We stress here, that all the fluxes are volume averaged, and the volume enclosed by the separatrix of the island is much smaller than the total volume of the box. The total electro-static heat flux is driven also outside the island and is, consequently, much larger when compared with the vortex mode.) The bottom panel of Fig. 3 shows the spatially averaged temperature gradients through the O-point of the island as a function of time. Further confirmation that the presence of the vortex has a profound effect on ion temperature gradient maintained within the island. The presence of the vortex, evidenced by an increased flux at that length scale, does indeed have the effect of reducing the gradient within the island. This effect is present in the electrons but less pronounced as the separation of time scales between parallel and perpendicular transport is more significant.

The vortex mode is strong in the LR cases, but has been found to be much reduced in the HR case. The reason for this is not entirely clear. It might be that the larger scale separation between turbulence and the island size of the HR case reduces the nonlinear pumping of the vortex mode. It might, however, also be related to the gradients in the island. The HR case has a ion temperature gradient above the linear threshold, and the nonlinear interaction with the driven instabilities could influence the evolution of the vortex mode.

The presence of the vortex mode also has an effect on the level of turbulence convection/spreading through the island O-point. Fig. 6 shows the fluctuation amplitudes through the O-point for simulation runs where the vortex is present (dashed lines) and where it is absent (solid lines). In the presence of a vortex the transition from the high turbulence outside the island to the suppressed level within the island is much sharper than in the case where no vortex is formed. The $1 /$ e length (exponential damping length) in the case where 

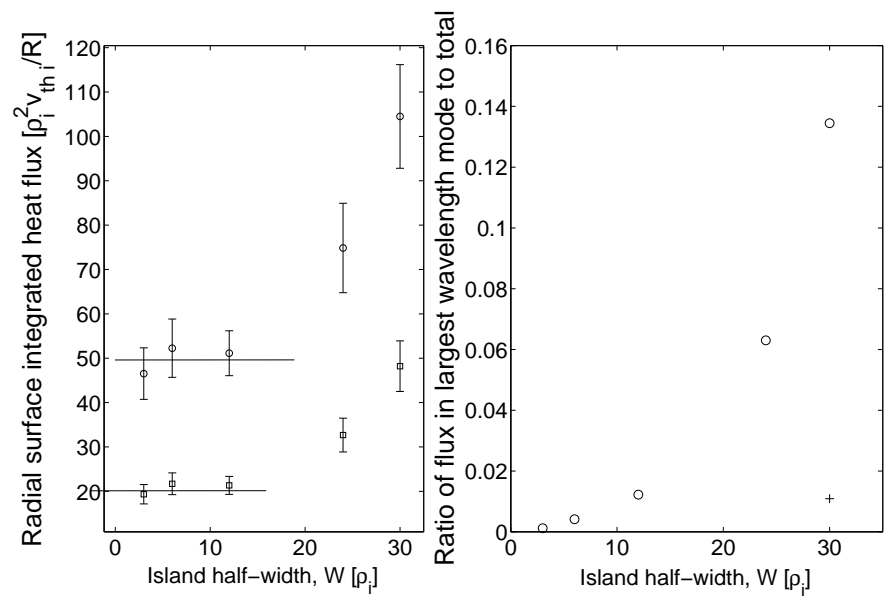

Figure 8: Left: The ion and electron heat fluxes for varying island widths showing a distinct increase in heat flux at large island sizes. Right: The ratio of ion heat flux due to large scale vortex mode compared with the total heat flux for kinetic runs (circles). Also shown is the heat flux when electrons are treated adiabatically, which, is widely different from the kinetic case. All data here was obtained from case LRa.

a vortex is present is $\sim 12 \rho_{*}$ whereas it is reduced to $\sim 2 \rho_{*}$ in the case with the vortex. The ExB flows associated with the vortex rapidly tear up the turbulent eddy structures as they enter the island. As it is the turbulence within the island that is responsible for perpendicular transport, the shearing of the eddies has the effect of reducing the perpendicular heat flow, and in turn, allowing for a more complete flattening of the temperature profile. This is most evident in the ion populations, which are compared in Fig. 6.

It is noted here, that the nonlinear pumping has previously been discussed in Ref. [30] which studies the linear stability of large scale modes in the presence of small scale turbulence. The vortex structure observed in our simulations, however, is different from the modes studied in Ref. [30] whose growth rates depend on the resistivity, which is zero in our collisionless simulations. Furthermore, our simulations have an island structure as initial condition. The large scale potential structures outside the island, but NOT the vortex structure inside the island, are also found in simulations without turbulence and represent the equilibrium flow connected with the island [14]. The strength of the convective cell is largely enhanced by the modified flow response around the island analogous to the generation of zonal flows.

\section{$5 \quad$ Finite electron temperature gradient and bootstrap current}

It is generally accepted that the fast transport along the magnetic field flattens the radial pressure gradient in the island, provided the perpendicular transport across the island is negligible against the parallel motion around the island [8]. For the timescales of our simulation we have previously estimated the electrons to have a zero temperature gradient inside the island due to their very rapid motion along the field line. The simulations, however, do not reproduce this effect as a finite electron temperature gradient is observed. 


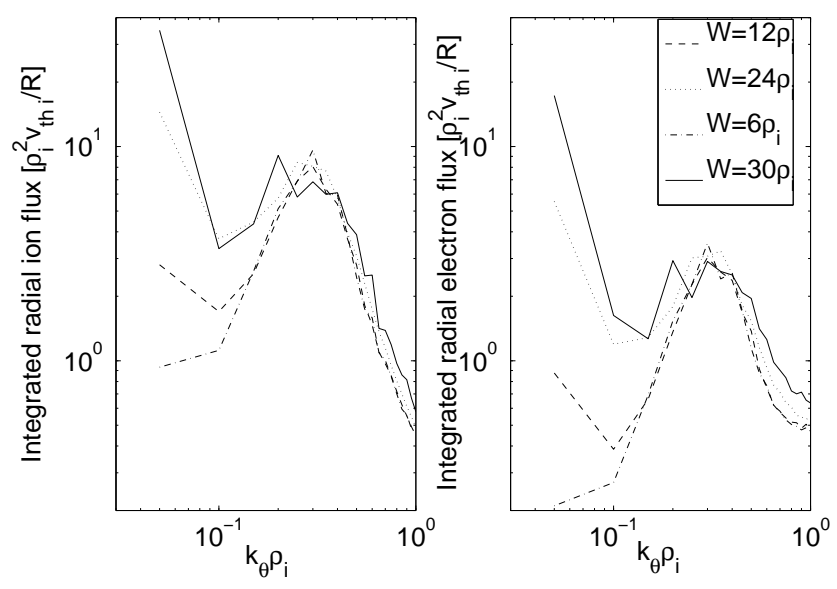

Figure 9: The contribution to the electrostatic heat flux from the different toroidal modes as a function of the normalized poloidal wave vector for different island sizes for case LRa. The left panel shows the spectra for ions and right shows the electron spectra for four different island widths.

The presented simulations uncover an overlooked element in the theoretical description of the NTM. While the passing electrons move along the field lines resulting in a radial profile flattening, the trapped electrons do not, since the field line length around the island is much longer than $2 \pi q R$. The trapped electron population can, therefore, maintain a radial temperature gradient despite the high thermal velocity of the electrons. This effect is verified through the data shown in Fig. 4 which shows time integrated (during statistical steady state) temperature profiles (background plus perturbed) along a radial chord through the O-point of the island at both the low as well as the high field side. These temperature profiles are determined by integrating the energy in the distribution function and consequently combine the effect of the trapped and passing population. However, since no trapped particles reach the high field side, the cord through the O-point on the high field side measures the temperature of the passing particles only. It can be observed that the latter profile indeed has a zero gradient. For passing particles the parallel heat transport dominates the solution leading to this zero gradient. The gradient on the low field side is finite, and its non-zero value is due to the trapped electrons only. In these simulations the trapped particle fraction is approximately $\sim \sqrt{\epsilon} \sim 0.5$. The reduction in the electron temperature gradient of roughly a factor two is consistent with the assumption that the trapped electrons show no profile flattening. The effect is not limited to the electrons, since the same physics processes determine the ion temperature gradient. Due to the separation of timescales, the effect is, however, more easily observed for the electrons.

We note here that the vortex mode can change the picture above. The $E \times B$ velocity does rotate the trapped particles around the island and can therefore lead to a reduction in the trapped particle gradient. Another effect, not included in our simulations, is the effect of collisions. The collisions will scatter particles from the trapped to the passing domain after which they will rapidly move around the island. Collisions can therefore reduce the radial gradient of the trapped population. Whether they will be effective in doing so will depend on the ratio of the collisional de-trapping time to the radial diffusive time. Estimating the 
diffusion time for turbulence to spread across the island

$$
\tau_{D_{s}}=w^{2} / 2 D_{s \perp}
$$

and using for the de-trapping rate

$$
\tau_{T s}=\epsilon / \nu_{s},
$$

one derives a critical island width $\left(w_{c s}\right)$ for species $s$ below which the collisions are ineffective, and a radial gradient in the trapped population can be maintained:

$$
w_{c s}=\sqrt{2 D_{s \perp} r / R \nu_{s}} .
$$

The observation made above has important consequences for the NTM. It is the gradient in the trapped population that is responsible for the bootstrap current (see Ref. [32] and the references cited therein). We therefore infer that the bootstrap current is not reduced inside the island in the collisionless limit, and the NTM would be stable in the latter case, even if the estimate of parallel to perpendicular transport would indicate a complete flattening. Since our simulations are collisionless, this conclusion can not be directly verified through a measurement of the bootstrap current as a diagnostic of the simulations. However, we have verified the statements made in this section using a reduced model in which both radial diffusion as well as collisions are kept. The results of this model will be published elsewhere[33].

\section{Conclusions}

In this paper we study the profile relaxation and transport processes in the proximity of an island structure in the magnetic field. Our numerical solution are based on the state of the art gyro-kinetic framework. The results have important implications for the neoclassical tearing mode theory. In particular, they treat more thoroughly the evolution of the gradients inside the island which are of importance for the bootstrap current drive, and can determine the non-linear stability threshold. The perpendicular heat transport which plays an important part in the determination of the radial gradients has, so far, been modelled with a uniform perpendicular heat diffusion coefficient. Our simulations show, perhaps not surprisingly, that this is a rather poor approximation. In particular, the following results have been obtained.

- Outside the island, the turbulent fluctuations are much larger at the X-point when compared with the O-point location. At the latter location flow shearing leads to a strong increase in the gradients, while the turbulent fluctuation level is not strongly enhanced.

- Inside the island, the reduced gradients lead to a reduction of the turbulent transport, and the local drive can completely vanish if the gradient falls below the threshold of the instability drive. The turbulent transport can, however, not be treated as a local problem since turbulence convection/spreading generates a finite fluctuation amplitude even in the absence of a local turbulent drive. A substantial part of the turbulence enters the island from the X-point moving in the drift direction. The latter turbulence convection is different from the diffusive picture discussed in the literature.

- The reduction of confinement due to the profile flattening inside the island is found to dominate over the turbulence reduction due to sheared plasma flows, and the net effect of the island is a reduction of confinement which increases with increasing island size. 
- Under some circumstances the turbulence can generate a large scale vortex mode through the nonlinear energy transfer from small to larger scales. This vortex is observed to coincide with the island and its strength is largely enhanced by the modified flow response connected with the perturbed magnetic field, in a way similar to the generation of the zonal flows in the case without island. Different from the zonal flow which do not transport heat, the vortex mode has a radial velocity component and the heat transport it generates can compete with the parallel streaming along the magnetic field.

- Even in the case in which the timescale for the transport parallel to the magnetic field line around the island is much smaller than the time scale for perpendicular transport across the island, a finite temperature gradient can remain inside the island. The latter effect is due to the trapped particles which do not move along the field line around the island. The radial gradient of the trapped particle population will be determined by ratio of the timescales for perpendicular transport and either the detrapping time or the timescale on which the vortex mode rotates the trapped particles around the island. Since the bootstrap current is driven by the radial gradient in the trapped particle population, the finite trapped particle gradient implies a non vanishing bootstrap current and therefore an absence of the neoclassical drive of the tearing mode.

The first two points confirm the results presented in Ref. [19]. However, the inclusion of electron dynamics has uncovered several important features, in particular the generation and time evolution of the vortex mode and its effect on the transport around the island, which was not seen in Ref. [19] and when the adiabatic approximation was used in GKW.

\section{Acknowledgments}

This work was jointly funded by the EPSRC via the S\&I grant and UKAEA/Euratom via Mobility. This work used resources on the HECToR supercomputer that were provided by the Engineering and Physical Sciences Research Council [grant number EP/H002081/1].

\section{References}

[1] H.P. Furth, J. Killeen, M.N. Rosenbluth, Phys. Fluids 6459 (1963)

[2] P.H. Rutherford, Phys. Fluids 161903 (1973)

[3] K. Ikeda, Nucl. Fusion 476 (2007)

[4] R. Carrera, R.D. Hazeltine, M. Kotschenreuther, Phys. Fluids 29899 (1986)

[5] Z. Chang, J.D. Callen, E.D. Fredrickson, R.V. Budny, C.C. Hegna, K.M. McGuire, M.C. Zarnstorff, and TFTR group, Phys. Rev. Lett. 7423 (1995)

[6] R.J. La Haye, Phys. Plasmas 13055501 (2006)

[7] H.R. Wilson, J.W. Connor, R.J. Hastie, and C.C. Hegna, Phys. Plasmas 3, 248 (1996)

[8] R. Fitzpatrick, Phys. Plasmas 2, 825 (1995)

[9] M. Muraglia, O. Agullo, S. Benkadda, X. Garbet, P. Beyer, and A. Sen, Phys. Rev. Lett. 103, 145001 (2009) 
[10] M. Muraglia, O. Agullo, M. Yagi, S. Benkadda, P. Beyer, X. Garbet, S.-I. Itoh, K. Itoh and A. Sen, Nucl. Fusion 49, 055016 (2009)

[11] F.L. Waelbroeck, F. Militello, R. Fitzpatrick and W. Horton, Plasma Phys. Contr. Fusion 51, 015015 (2009)

[12] A. Ishizawa, N. Nakajima, Nucl. Fusion 47, 1540-1551 (2007)

[13] S.-I. Itoh, Plasma Phys. Contr. Fusion 46, 123 (2004)

[14] H.R. Wilson, and J.W. Connor, Plasma Phys. Contr. Fusion 51, 115007 (2009)

[15] F.L. Waelbroeck, Nucl. Fusion 49104025 (2009)

[16] H. Biglari, P.H. Diamond, and P.W. Terry, Phys. Fluids B 21 (1990)

[17] R.E. Waltz, G.B. Kerbel, and J. Milovich, Phys. Plasmas 1, 2229 (1994)

[18] E. Joffrin, C.D. Challis, G.D. Conway, X. Garbet, A. Gude, S. Gnter, N.C. Hawkes, T.C. Hender, D.F. Howell, G.T.A. Huysmans, E. Lazzaro, P. Maget, M. Marachek, A.G. Peeters, S.D. Pinches, S.E. Sharapov and JET-EFDA contributors, Nuclear Fusion 43, 1167 (2003)

[19] E. Poli, A. Bottino and A.G. Peeters, Nucl. Fusion 49, 075010 (2009)

[20] A.G. Peeters, D. Strintzi, Phys. Plasmas 11, 3748 (2004)

[21] A.G. Peeters, Y. Camenen, F.J. Casson, W.A. Hornsby, A.P. Snodin, D. Strintzi, and G. Szepesi, Comp. Phys. Comm. 180, 2649 (2009)

[22] S. Hamada, Kakuyugo Kenkyu 1, 542 (1958)

[23] A.M. Dimits, G. Bateman, M.A. Beer, B.I. Cohen, W. Dorland, G W. Hammett, C. Kim, J.E. Kinsey, M. Kotschenreuther, A.H. Kritz, L.L. Lao, J. Mandrekas, W.M. Nevins, S.E. Parker, A.J. Redd, Phys. Plasmas 7, 3 (2000)

[24] ASDEX Upgrade Team, Nucl. Fusion 391321 (1999)

[25] X. Garbet, Phys. Plasmas 8, 2792 (2001)

[26] X. Garbet, R.E. Waltz, Phys. Plasmas 3, 5 (1996)

[27] Z. Lin, Phys. Plasmas 11, 1099 (2004)

[28] V. Naulin, Phys. Plasmas 12, 122306 (2005)

[29] T S Hahm, P H Diamond, Z Lin, K Itoh and S-I Itoh, Plasma Phys. Control. Fusion 46 A323 (2004)

[30] C.J. McDevitt, P.H. Diamond, Phys. Plamsas 13, 032302 (2006)

[31] R.E. Waltz, J. Candy, Phys. Plasmas 12, 072303 (2005)

[32] A.G. Peeters, Plasma Phys. Control. Fusion 42 B231 (2000)

[33] W.A. Hornsby, A.G. Peeters, Interaction of turbulence with magnetic islands: Effect on bootstrap current. Phys. Plasmas to be submitted 